\title{
Quantitative TEM Tensile Testing on 1D Nano-structural Materials
}

\author{
Z. W. Shan*, J. Oh*, A. M. Minor**, S. A. S. Asif* and O. L. Warren* \\ * Hysitron, Inc. 10025 Valley View Road, Minneapolis, MN 55344 \\ ** National Center for Electron Microscopy, Lawrence Berkeley National Laboratory, Berkeley, \\ CA 94720
}

In situ transmission-electron-microscopy (TEM) tensile testing has been a powerful tool for revealing the underlying physical mechanism when materials are subjected to a stress [1-5]. With this technique, the dynamics microstructure evolution of the materials can be recorded in a nano or even atomic scale. However, all the commercial in situ TEM tensile holders available so far suffer from the absence of quantitative ability and the complexity in sample preparation.

Consequently, the potential exploration ability of in situ TEM tensile holders has been hindered substantially. Supported by Department of Energy (DOE) Small Business Innovation Research (SBIR) program, recently we have developed a new tensile device for operation inside a TEM which not only yields quantitative load-displacement data concomitant with real time images of the microstructural behavior, but also simplifies the sample preparation procedure essentially.

In this work, we report the current progress in the application of this quantitative in situ TEM tensile device for measuring the mechanical properties of 1D nanostructures (silicon nanowires etc). Fig. 1 is the TEM images taken after the fracture of silicon nanowire. It was surprised to see that apparent plasticity has occurred even for nano wire with a diameter of $250 \mathrm{~nm}$. As shown in Fig. 1a, three zones can be identified along the residual nano wire based on the contrast. Zone I corresponds to area without plastic deformation. Zone III represents area that has experienced heavy plastic deformation. Zone II, bounded by red and green dash line, indicates gradually decrease of plastic deformation from Zone 3 to Zone 1. The insets at the upper left corner and lower right corner are corresponding selected area different patterns from zone III and zone I, respectively. Besides the slight relative change of the density of second diffraction spots (Marked by white arrow in the insets of Fig. 1 a), there are no indication for either amorphous or polycrystallization. Fig. $1 \mathrm{~b}$ is the magnified dark field image of zone II and III shown in Fig. 23a. Dislocation like contrast can be identified clearly. The findings indicate new deformation mechanism for silicon nano wires which has been seen as classic brittle material in bulk form.

References

[1] Z. W. Shan et al., Science 305, 654 (JUL 30, 2004).

[2] Z. W. Shan et al., Physical Review Letters 98 (Mar, 2007).

[3]. Z. W. Shan et al., Phys. Rev. Lett 100, 105502 (2008).

[4] Y. Zhu, C. Ke, H. D. Espinosa, Experimental Mechanics 47, 7 (Feb, 2007).

[5] J. Rajagopalan, J. H. Han, M. T. A. Saif, Science 315, 1831 (Mar, 2007)

[6] This work was supported by a DOE SBIR Phase I\&II grant (DE-FG02-07ER84813)

awarded to Hysitron, Inc., which does not constitute an endorsement by DOE of the views expressed in this article. Research performed at the National Center for Electron Microscopy was supported by the Scientific User Facilities Division of the Office of Basic Energy Sciences, U.S. Department of Energy under Contract \# DE-AC02-05CH1 1231. 


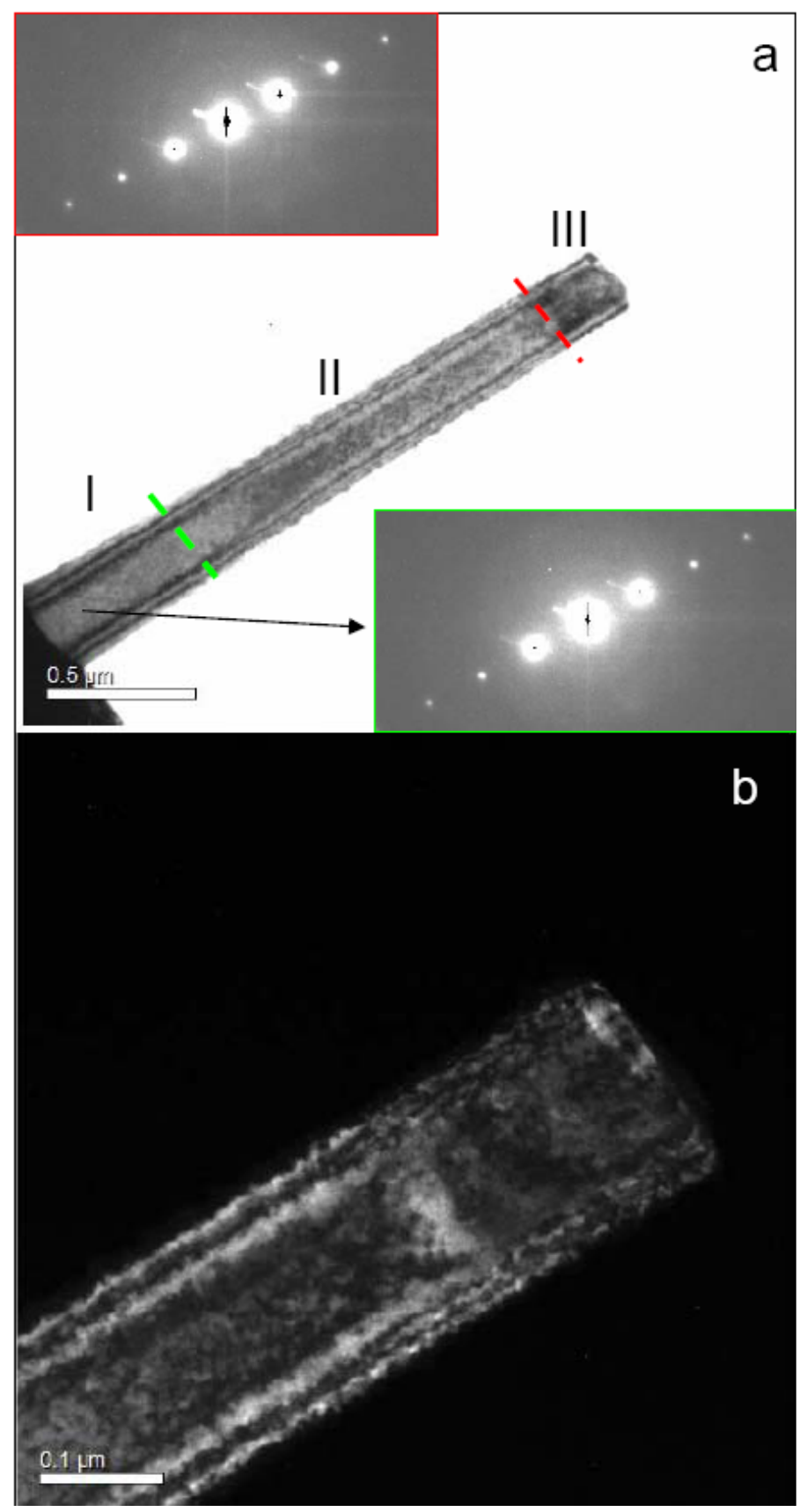

Fig. 1. Postmortem TEM observation of uniaxial tensile fractured Si nano wire. (a) Three zones can be identified along the residual nano wire based on the contrast. The insets at the upper left corner and lower right corner are corresponding selected area different patterns from zone III and zone I, respectively. (b) Magnified dark field image of zone II and III shown in Fig. 1a. Dislocation like contrast can be identified clearly [6]. 